Ventilatortourenzahl 1490 pro Minute,

Eintrittsstutzen-Vakuum $93 \mathrm{~mm}$, Temperatur 23, Austrittsstutzen-Druck $142 \mathrm{~mm}$, Temperatur $25^{\circ}$,

'T'emperatur vor Hahnmesser $30^{\circ}$,

Hahnmesserstellung links 7, Druckdifferenz $450 \mathrm{~mm}$,

Austrittsstutzenleitung $450 \mathrm{Durchm}$, gedrosselt durch den Meßschieber auf $96500 \mathrm{qmm}$, wobei 2,6 $\mathrm{mm}$ Druckdifferenz entstanden,

Baroneterstand $730 \mathrm{~mm}$,

Gasaralysen vor der Austrittsdrosselung ergaben im Mittel 0,7425 Proz. schweflige Säure, nach der Keichschen Methode mittels itoo N.-Jodlösung bestimmt.

Zeitdauer für die Zuführung der $20 \mathrm{~kg}$ schwefliger S:iture 23 Minuten 6 Sekunden.

Mithin entstand folgende Rechnung:

$1 \mathrm{cbm} \mathrm{SO}$, wiegt bei $25^{\circ}$ und $730 \mathrm{~mm} \mathrm{~B}$.

$$
1+0,00366 \cdot 25 \cdot 760=2,525 \mathrm{~kg} \text {. }
$$

Verbrautht wurden $20 \mathrm{~kg}=17,92 \mathrm{cbm}$; diese waren vorhanden in $0,742 \vec{\jmath}$-proz. Form, mithin in 1067,4 cbm in 23 Min. 6 Sek. Mithin sind gefürlert pro Minute $46,2 \mathrm{cbm}$.

Ein Kontrollversuch ergab folgende Zahlen: Ventilatortourenzuhl 1460 pro Minute,

Eintrittsstutzen-Vakuum $93 \mathrm{~mm}$, Temperatur $23^{\circ}$ Austrittsstutzen-Druck $142 \mathrm{~mm}$, Temperatur $25^{\circ}$,

Temperatur vor Hahumesser $30^{\circ}$,

Hahnmesserstellung links 7, Druckdifferenz $375 \mathrm{nmm}$ A ustrittsstutzenleitung 450 Durchm., ged rosselt durch den Meßschiober auf $96500 \mathrm{qmm}$, wobei $2,5 \mathrm{~mm}$ Druckclifferen\% entstanden,

Barometerstund $730 \mathrm{~mm}$,

Gasanalysen ergaben im Mittel 0,692 Proz. $\mathrm{SO}_{2}$,

Zeitdauer füu die Zuführung der $20 \mathrm{~kg}$ schwefliger Săure 2; Minuten 17 Sekunden.

Hieruus berechnet ergaben sich $45,3 \mathrm{cbm}$ Gas pro Minute. Bei 1490 Touren würden $46 \mathrm{cbm}$ heralskomunen.

Man sieht hieraus, dab die Resultate sich in genügender Übereinstimmung halten.

Wäre das Meßgas einem in die Apparatur eingebauten Ventilator zugefülurt worden, so hätte man sein Volumen abzuziehen von dem gefundenen Gasquantum. Wird die Messung mit einem Gase verschiedenen spezifischen Gewichts gemacht, so ist dies zu berücksichtigen, so wird z. B. bei sonst gleichen Verhältnissen ein Gas mit dem doppelten spezifischen Gewicht nur in halber Menge gefördert.

Es braucht wohl nicht erst darauf hingewiesen $\mathrm{zu}$ werden, daß die gleiche MeBmethode auch für Flüssigkeiten anwendbar ist, mögen diese in offenen oder geschlossenen Leitungen fließen, wenn man ein gemessenes Quantum einer "Meßflüssigkeit" in konstantem Strome sich mit ihr vermischen läßt und das Gemisch analysiert. Letztere Methode erinnert an das Verfahren von Dr. Perl und Kur (Chem.-Ztg. 1888, S. 1109) zur Volumenbestimmung von Gefäßen.

\section{Zwischenreaktionen. Von A. Skrabal.}

Dnter obigem Titel brachte Herr Riedel ${ }^{1}$ ) eine Abhandlung, in welcher er sich gegen die Idee des "beweglichen Gleichgewichtes" und die zur Erklärung gewisser katalytischer Vorgānge aufgestellte, "Theorie der Zwischenreaktionen " wendete.

Herr Riedel betrachtet das bewegliche Gleichgewicht „Flüssiglseit-Dampf", welches darauf fuBt, daB in der Zeiteinheit ebensoviel Flüssigkeit verdampft, als Dampf niedergeschlagen wird, und sagt: "Nach-thermodynamischer Denkweise repräsentiert aber das Gebilde ein Perpetuum mobile. Wenn ein Stoff freiwillig aus dem Zustande $\mathbf{A}$ in den Zustand B übergeht, so vermehrt sich seine Entropie. Der umgekehrte Vorgang müBte dann von einer Entropieverminderung begleitet sein. Das ist hier ausgeschlossen, da er ja von selbst eintreten soll, Vorgänge dieser Art aber nur unter Entropievermehrung vor sich gehen können". Nun ist aber zu bedenken, daß die beiden entgegengesetzten Vorgänge voneinander nicht unabhängig, nicht hintereinander, sondern nebeneinander verlaufen, und daB im Falle des Gleichgewichtes zufolge des einen Vorganges die Entropie in demselben MaBe wächst, als sie zufolge des anderen abnimmt, daß also - das charakteristische Kennzeichen des Gleichgewichtes - nach jedern Augenblicke die algebraische Summe der Entropieveränderungen gleich Null ist. Ein derartiges Gleichgewicht steht nicht im Widerspruche mit den Gesetzen der Energetik, ebensowenig wie ein reversibler KreisprozeB, bei welchem auch nach jedem Augenblicke die algebraische Summe der Entropieveränderungen Null ist und welcher ebenfalls nichts anderes ist als die kontinuierliche Aufeinanderfolge von verschiedenen Gleichgewichtslagen. Das bewegliche Gleichgewicht repräsentiert daher $k e$ in Perpetuum mobile, und die Thermodynamik spricht nicht gegen die Möglichkeit desselben.

Wenn aber eine Theorie, sei sie nun diejenige des "beweglichen Gleichgewichtes" oder irgend eine andere, mit den auf induktivem Wege gefundenen Gresetzen nicht im Widerspruche steht und anderseits viele Erscheinungen "plausibel " macht, so hat sie den Beweis ihrer Existenzberechtigung erbracht - mehr wird von keiner Theorie verlangt -- und braucht erst dann fallen gelassen zu werden, bis eine neuere und bessere aufgestellt worden ist, was in

1) Diese Zeitschr. 1903, Heft 21, S. 492. 
unserem Falle nachweislich noch nicht zutrifft.

Ferner wendet sich Herr Riedel gegen die katalytische Theorie der $\mathbf{Z w i s c h e n r e a k - ~}$ tionen, welche ebenfalls im Widerspruche mit der Thermodynamik sein soll. Auch diese Behauptung ist nicht zutreffend. Ist ein arbeitsfähiges System gegeben, so sagen zwar die Hauptsätze, daB ein Vorgang eintreten $k a n n$, nicht aber, daB er eintreten muB, noch sagen sie über die Geschwindigkeit des Vorganges etwas Bestimmtes aus. Die Hauptsätze werden daher auch nicht verletzt, wenn durch irgend einen Umstand, durch welchen der Energiegehalt des Systemes nur unerheblich verändert wird, die Geschwindigkeit des Vorganges merklich vergrößert erscheint. Ein derartiger Umstand kann nun ganz wohl die Gegenwart eines Katalysators sein, wenn letzterer durch geschwinder verlaufende $Z$ wischenreaktionen einen neuen Weg schafft, auf welchem die Abnahme der freien Energie " des gegebenen Systemes rascher erfolgen kann. Betrachten wir die Reaktion

$$
\mathrm{A}+\mathrm{B}=\mathrm{AB} \text {. }
$$

so wird der Vorgang offenbar dann beschleunigt vor sich gehen, wenn, trotz der geringen Konzentration des Katalysators $\mathrm{K}$, die beiden Reaktionen

$$
\mathrm{A}+\mathrm{K}=\mathrm{AK} \text {. }
$$

und

$$
\mathrm{AK}+\mathrm{B}=\mathrm{AB}+\mathrm{K} \text {. }
$$

in Summe rascher verlaufen als die Reaktion (1). Allerdings muß, was Ostwald betont, der raschere Verlauf der beiden Zwischenreaktionen (2) und (3) durch Messung festgestellt werden, damit der vollgültige Beweis erbracht erscheint. Es werden auf jeden Fall alle drei Reaktionen gleichzeitig verlaufen, häufig wird aber der Umsatz nach (2) und (3) so rasch erfolgen, daB wir von dem Umsatz nach Reaktion (1) absehen können.

Herr Riedel stützt sich ganz besonders auf die Autorität Ostwalds, welch Letzterer mit Nachdruck auf die Unwahrscheinlichkeit, welche die Theorie der $\mathrm{Z}$ wischenreaktionen enthālt, hingewiesen haben soll. DaB Ostwald nicht dieser Ansicht ist, glaube ich aus seinem Vortrag "Über Katalyse" ") entnehmen zu können. Ostwald sträubt sich weniger gegen diese Theorie, als gegen den Unfug, welcher mit ihr getrieben wird, und welcher entweder darin besteht, daß man alle Katalysen im Sinne der Zwischenreaktionentheorie zu erklären sucht, oder dab man ihre Gültigkeit annimmt, ohne den 1901

2) 73. Naturforscherversammlang in Hamburg, unter den vorhandenen Bedingungen rascheren Verlauf der $Z$ wischenreaktionen gemessen zu haben.

Es ist daher über die "Theorie der Zwischenreaktionen" dasselbe wie über die Idee des „beweglichen Gleichgewichtes ${ }^{\text {z }}$ zu sagen.

Wien, am 4. Juni 1903.

\section{Über die Frage nach der „Konstitution des Portland-Zementes. \\ Von Privatdozent Dr. P. Rohland.}

Zunächst möchte ich mir gestatten, auf eine Bemerkung von E. Jordis und E. Canter in ihrer Abhandlung über „die geschichtliche Entwicklung der Theorien über die Konstitution von Portland-Zement ${ }^{1}$ ) zurūckzukommen. Dieselben haben nämlich einen Satz aus dem Auszuge meiner Antrittsvorlesung über „den Einflub der physikalisch-chemischen Gesetze auf einige Aufgaben und Probleme der anorganischen Chemie ${ }^{2}$ ) herausgegriffen und offenbar in dem Sinne $\mathrm{nich} t$ aufgefaßt, wie er aufgefußt werden muß.

Dieser Satz lautet wörtlich:

"Auf Reaktionen zwischen Stoffen im festen Aggregatzustande mit sehr weringer Geschwindigkeit beruhen die Vorgänge im erhărtenden Portland-Zement; die Leistungsfähigkeit ein und desselben Produktes ist in vielen Fällen von dem Grade seiner mehr oder weniger feinen Zerteilung abbängig; es ist bereits erwiesen ${ }^{3}$ ), $\mathrm{dab}$ Zementkörner, welche ein Sieb von 900 Maschen auf $1 \mathrm{qcm}$ nicht mehr passizren können, für die Hydratations- und Erhärtungsreaktion nicht in Betracht kommen."

Schon die Worte: "mit sehr geringer Geschwindigkeit" und weiterhin , für die Hydratationsund Erhärtungsreaktion “ hätten auf die richtige. Bedeutung des Satzes führen können, denn einmal existieren Portland-Zemente mit sehr verschieden großer Hydratationsgeschwindigkeit; and ferner beziehen sich selbstrerständlich diese Reaktionen zwischen Stoffen in der festen Formart gar nicht auf die HydratationsphasedesPortland-Z omentes, in welcher der Betrag der Hydratationsgeschwindigkeit eben ein sehr variabler sein kann - es hängt das mit dem Werte der Lösungstension des Portland-Zementes dem Wasser und elektrolytischen Salzlōsungen, welche zugleich nkatalytische" sind, gegenūber zusammen (s. weiter unten), sondern auf die sich daran schließende Erhärtungsperiode, während welcher nach der Wasserbindung durch das in fester Lösung mit den siliciumbaltigen Bestandteilen des Portland-Zementes befindliche Calciumoxyd und die letzteren nebenher die

1) Diese Zeitschrift 1903, No. 21.

2) Diese Zeitschrift 1903, No. 11.

3) Büsing und Schumann, der Portland-Zement und seine Anwendung im Bauwesen. 2. Aufl. 